PROCEEDINGS OF THE

AMERICAN MATHEMATICAL SOCIETY

Volume 127, Number 8, Pages 2335-2342

S 0002-9939(99)04779-6

Article electronically published on April 9, 1999

\title{
A FIXED POINT THEOREM AND ITS APPLICATION TO INTEGRAL EQUATIONS IN MODULAR FUNCTION SPACES
}

\author{
A. AIT TALEB AND E. HANEBALY \\ (Communicated by Dale Alspach)
}

\begin{abstract}
In this paper we present a fixed point theorem of Banach type in modular space. We give an application of this result to a nonlinear integral equation in Musielak-Orlicz space.
\end{abstract}

\section{INTRODUCTION}

It is well known that one of the standard proofs of Banach's fixed point theorem is based on Cantor's theorem in complete metric spaces [3, 4]. To this end, using some convenient constants in the contraction assumption, we present a generalization of Banach's fixed point theorem in some classes of modular spaces, where the modular is $s$-convex, having the Fatou property and satisfying the $\Delta_{2}$-condition.

As an application we study the existence of a solution for an integral equation of Lipschitz type in a Musielak-Orlicz space.

We begin by recalling some basic concepts of modular spaces; for more information, we refer to the books by Musielak [8] and Kozlowski [7].

Definition 0-1. Let $X$ be an arbitrary vector space over $K(=\mathbf{R}$ or $\mathbf{C})$.

a) A functionnal $\rho: X \rightarrow[0,+\infty]$ is called modular if:

i) $\rho(x)=0 \Longleftrightarrow x=0$.

ii) $\rho(\alpha x)=\rho(x)$ for $\alpha \in K$ with $|\alpha|=1, \forall x \in X$.

iii) $\rho(\alpha x+\beta y) \leq \rho(x)+\rho(y)$ if $\alpha, \beta \geq 0, \alpha+\beta=1, \forall x, y \in X$.

b) If iii) is replaced by:

iii' $^{\prime} \rho(\alpha x+\beta y) \leq \alpha^{s} \rho(x)+\beta^{s} \rho(y)$ for $\alpha, \beta \geq 0, \alpha^{s}+\beta^{s}=1$ with an $\left.\left.s \in\right] 0,1\right]$, then the modular $\rho$ is called an $s$-convex modular; and if $s=1, \rho$ is called convex modular.

c) A modular $\rho$ defines a corresponding modular space, i.e. the space $X_{\rho}$ given by

$$
X_{\rho}=\{x \in X \mid \rho(\lambda x) \rightarrow 0 \text { as } \lambda \rightarrow 0\} .
$$

Remarks. 1) Note that in general there is no reason to expect the subadditivity of a modular $\rho$. Nevertheless, in view of iii) from Definition 0-1 the inequality $\rho(x+y) \leq \rho(2 x)+\rho(2 y)$ holds.

Received by the editors January 5, 1996 and, in revised form, October 30, 1997.

1991 Mathematics Subject Classification. Primary 46A80, 47H10, 45G10, 46E30.

Key words and phrases. Modular space, fixed point, integral equation.

(C)1999 American Mathematical Society 
2) If $\rho$ is convex modular, the modular space $X_{\rho}$ can be equipped with a norm called the Luxemburg norm defined by:

$$
|x|_{\rho}=\inf \left\{\alpha>0 ; \rho\left(\frac{x}{\alpha}\right) \leq 1\right\} .
$$

3) As a classical example, we would like to mention the Musielak-Orlicz space denoted by $L^{\varphi}[8]$ and the modular function space denoted by $L^{\rho}[7]$.

Definition 0-2. Let $X_{\rho}$ be a modular space.

a) A sequence $\left(x_{n}\right)_{n}$ in $X_{\rho}$ is said to be:

i) $\rho$-convergent to $x$ if $\rho\left(x_{n}-x\right) \rightarrow 0$ as $n \rightarrow+\infty$.

ii) $\rho$-Cauchy if $\rho\left(x_{n}-x_{m}\right) \rightarrow 0$ as $n$ and $m \rightarrow+\infty$.

b) $X \rho$ is $\rho$-complete if any $\rho$-Cauchy sequence is $\rho$-convergent.

c) A subset $B \subset X_{\rho}$ is said to be $\rho$-closed if for any sequence $\left(x_{n}\right)_{n} \subset B$ with $x_{n} \rightarrow x$, then $x \in B$. $\bar{B}^{\rho}$ denotes the closure of $B$ in the sense of $\rho$.

d) A subset $B \subset X_{\rho}$ is called $\rho$-bounded if:

$\delta_{\rho}(B)=\sup _{x, y \in B} \rho(x-y)<+\infty$, where $\delta_{\rho}(B)$ is called the $\rho$-diameter of $B$.

e) We say that $\rho$ has the Fatou property if:

$$
\rho(x-y) \leq \liminf \rho\left(x_{n}-y_{n}\right)
$$

whenever

$$
x_{n} \stackrel{\rho}{\rightarrow} x \text { and } y_{n} \stackrel{\rho}{\rightarrow} y .
$$

f) $\rho$ is said to satisfy the $\Delta_{2}$-condition if: $\rho\left(2 x_{n}\right) \rightarrow 0$ as $n \rightarrow+\infty$ whenever $\rho\left(x_{n}\right) \rightarrow 0$ as $n \rightarrow+\infty$.

\section{FiXeD POINT THEOREM}

I-1. Theorem I-1. Let $X_{\rho}$ be a $\rho$-complete modular space. Assume that $\rho$ is an $s$-convex modular satisfying the $\Delta_{2}$-condition and having the Fatou property. Let $B$ be a $\rho$-closed subset of $X_{\rho}$ and $T: B \rightarrow B$ a mapping such that:

$$
\exists c, k \in \mathbf{R}^{+}: c>\max (1, k), \rho(c(T x-T y)) \leq k^{s} \rho(x-y) \forall x, y \in B .
$$

Then $T$ has a fixed point.

Remarks. 1) It is natural to introduce the constants $c$ and $k$ in the assumption of strict contraction in modular spaces. Note also that Theorem I-1 and its proof become more simple in the particular case where $s=1(\rho$ is convex ) and $c=$ $2>k>0$; see [1].

2) The contraction $(*)$ in Theorem I-1 is also true for any constant $c_{0}$ such that $1<c_{0} \leq c$ :

$$
\begin{aligned}
\rho\left(c_{0}(T x-T y)\right) & =\rho\left(\frac{c_{0}}{c} c(T x-T y)\right) \\
& \leq \frac{c_{0}^{s}}{c^{s}} \rho(c(T x-T y)) \leq k_{0}^{s} \rho(x-y)
\end{aligned}
$$

where $k_{0}=\frac{c_{0}}{c} k<c_{0}$, since $\frac{k}{c}<1$. 
I-2. Proof of Theorem I-1. $1^{\text {st }}$ step: The proof of Theorem I-1 is based on the next result which is the modular formulation of Cantor's theorem:

Theorem I-2. Let $X_{\rho}$ be a $\rho$-complete modular space. Let $\left(F_{n}\right)_{n}$ be a decreasing sequence of nonempty, $\rho$-closed subset of $X_{\rho}$ with $\delta_{\rho}\left(F_{n}\right) \rightarrow 0$ as $n \rightarrow+\infty$. Then $\bigcap_{n} F_{n}$ is reduced to one point.

The proof of this result uses the same ideas as in complete metric spaces and it suffices to replace the distance by the modular $\rho$.

$2^{\text {nd }}$ step. Let $\left(\epsilon_{n}\right)_{n}$ be a decreasing sequence of positive numbers such that $\epsilon_{n} \rightarrow 0$ as $n \rightarrow+\infty$, and consider the sets defined by:

$$
M_{\epsilon_{n}}=\left\{x \in B \mid \rho(L(x-T x)) \leq \epsilon_{n}\right\},
$$

where $L=\max \{c, 2 \alpha\}$ and $\alpha$ is the $s$-conjugate of $c$, i.e. $\frac{1}{c^{s}}+\frac{1}{\alpha^{s}}=1$. Then the sequence $\left(M_{\epsilon_{n}}\right)$ has the following property:

1) $M_{\epsilon_{n}} \neq \emptyset, \forall n$.

We assume without any loss of generality that: $\exists x \in B$ such that $\rho(x-T x)<$ $+\infty$; then for $p \in \mathbf{N}^{*}$ we have:

$$
\begin{aligned}
& \rho\left(c\left(T^{p+1} x-T^{p} x\right)\right) \leq k^{s} \rho\left(T^{p} x-T^{p-1} x\right) \\
& \quad=k^{s} \rho\left(\frac{1}{c} c\left(T^{p} x-T^{p-1} x\right)\right) \\
& \quad \leq\left(\frac{k^{s}}{c^{s}}\right) k^{s} \rho\left(T^{p-1} x-T^{p-2} x\right),
\end{aligned}
$$

and by induction we deduce:

$$
\rho\left(c\left(T^{p+1} x-T^{p} x\right)\right) \leq\left(\frac{k}{c}\right)^{s(p-1)} \rho(x-T x) .
$$

Since $\frac{k}{c}<1$, we have $\rho\left(c\left(T^{p+1} x-T^{p} x\right)\right) \rightarrow 0$ as $p \rightarrow+\infty$. Thus by $\left(\Delta_{2}\right)$

$$
\rho\left(L\left(T^{p+1} x-T^{p} x\right)\right) \rightarrow 0 \quad \text { as } p \rightarrow+\infty .
$$

Hence: $\exists q \in \mathbf{N}^{*}$ such that $\rho\left(L\left(T^{q+1} x-T^{q} x\right)\right) \leq \epsilon_{n}$. Then: $y=T^{q} x \in M_{\epsilon_{n}}$.

2) $M_{\epsilon_{n}}$ is $\rho$-closed.

Let $\left(x_{p}\right)$ be a sequence in $M_{\epsilon_{n}}$. Assume that $\left(x_{p}\right)_{p}$ is $\rho$-convergent to $x \in X_{\rho}$, $\left(x_{p}\right)_{p} \subset B$ and $B$ is $\rho$-closed; it follows that $x \in B$.

$\rho\left(x_{p}-x\right) \rightarrow 0$ as $p \rightarrow+\infty$, by $\left(\Delta_{2}\right), \rho\left(L\left(x_{p}-x\right)\right) \rightarrow 0$ as $p \rightarrow+\infty$.

On the other hand $\rho\left(c\left(T x_{p}-T x\right)\right) \leq k^{s} \rho\left(x_{p}-x\right) \leq k^{s} \rho\left(L\left(x_{p}-x\right)\right)$; then

$$
\rho\left(c\left(T x_{p}-T x\right)\right) \rightarrow 0 \text { as } p \rightarrow+\infty .
$$
that:

Again by $\left(\Delta_{2}\right) \rho\left(L\left(T x_{p}-T x\right)\right) \rightarrow 0$ as $p \rightarrow+\infty$. The Fatou property implies

$$
\rho(L(T x-x)) \leq \liminf \rho\left(L\left(T x_{p}-x_{p}\right)\right) \leq \epsilon_{n} .
$$

Therefore $x \in M_{\epsilon_{n}}$ and hence $M_{\epsilon_{n}}$ is $\rho$-closed.

3) $\delta_{\rho}\left(M_{\epsilon_{n}}\right) \rightarrow 0$ as $n \rightarrow+\infty$.

Let $x, y \in M_{\epsilon_{n}}$; we have:

$$
\rho(x-y)=\rho\left(\frac{\alpha(x-T x)}{\alpha}+\frac{c(T x-T y)}{c}+\frac{\alpha(T y-y)}{\alpha}\right)
$$


where

$$
\begin{aligned}
\alpha^{s} & =\frac{c^{s}}{c^{s}-1} \\
& \leq \frac{1}{\alpha^{s}} \rho(\alpha(x-T x)+\alpha(T y-y))+\frac{1}{c^{s}} \rho(c(T x-T y)) \\
& \leq \frac{1}{2^{s} \alpha^{s}} \rho(2 \alpha(x-T x))+\frac{1}{2^{s} \alpha^{s}} \rho(2 \alpha(T y-y))+\frac{1}{c^{s}} \rho(c(T x-T y)) \\
& \leq \frac{1}{2^{s} \alpha^{s}}(\rho(L(x-T x))+\rho(L(T y-y)))+\frac{1}{c^{s}} \rho(c(T x-T y)) .
\end{aligned}
$$

Then

Hence,

$$
\rho(x-y) \leq \frac{1}{2^{s} \alpha^{s}} 2 \epsilon_{n}+\frac{k^{s}}{c^{s}} \rho(x-y) .
$$

and we deduce that

$$
\rho(x-y) \leq \frac{1}{2^{s} \alpha^{s}} 2 \epsilon_{n} \frac{c^{s}}{c^{s}-k^{s}}
$$

$$
\delta_{\rho}\left(M_{\epsilon_{n}}\right)=\sup _{x, y \in M_{\epsilon_{n}}} \rho(x-y) \leq \epsilon_{n} 2^{1-s} \frac{c^{s}-1}{c^{s}-k^{s}},
$$

and

$$
\delta_{\rho}\left(M_{\epsilon_{n}}\right) \rightarrow 0 \text { as } n \rightarrow+\infty .
$$

4) $\left(M_{\epsilon_{n}}\right)$ is decreasing. This is an immediate consequence of the fact that $\left(\epsilon_{n}\right)$ is decreasing.

It follows that the conditions of Cantor's theorem are satisfied and hence we have: $\bigcap_{n} M_{\epsilon_{n}}=\left\{x_{0}\right\}$.

But $x_{0} \in M_{\epsilon_{n}} \forall n \Rightarrow \rho\left(L\left(x_{0}-T x_{0}\right)\right) \leq \epsilon_{n} \rightarrow 0$ as $n \rightarrow+\infty \Rightarrow T x_{0}=x_{0}$.

Remark. We are unable to prove whether the conclusion of Theorem I-1 is true if we have $c=1$ and $0<k<1$. To this end, recall the following results by Khamsi-Kozlowski-Reich:

Theorem I-3 ([5]). Let $\rho$ be a modular function satisfying the $\Delta_{2}$-condition and let $B$ be a $|\cdot|_{\rho}$-closed subset of $L_{\rho}$.

$T: B \rightarrow B$ is a mapping such that: $\exists 0<k<1$ such that

$$
\rho(T f-T g) \leq k \rho(f-g) \quad \forall f, g \in B .
$$

Then: $T$ has a fixed point if one of the following assumptions is satisfied:

i) $\exists f_{0} \in B ; \sup _{n}\left(2 T^{n} f_{0}\right)<\infty$.

ii) $B$ is $\rho$-bounded.

If $i i$ ) is satisfied, the fixed point is unique. Note also that the modular $\rho$ in Theorem I-3 has the Fatou property and the $\Delta_{2}$-condition as in Theorem I-1. Also by $\left(\Delta_{2}\right)$ we have: $B$ is $\rho$-closed $\Leftrightarrow B$ is $|\cdot| \rho^{- \text {closed. }}$

On the other hand the strict inequality $\left(^{*}\right)$ in Theorem I-1 implies the inequality of Theorem I-3

$$
\rho(T x-T y)=\rho\left(\frac{1}{c}(c(T x-T y))\right) \leq \frac{1}{c^{s}} \rho(c(T x-T y)) \leq \frac{k^{s}}{c^{s}} \rho(x-y) .
$$

Consequently with some reinforced assumptions in Theorem I-1, namely the $s$ convexity of $\rho$ and the strict contraction $(*)$, we prove the existence of a fixed point 
for $T$ without restrictive conditions concerning the domain of $T$ : i) or ii) in Theorem I-3 .

The following example shows that Theorem I-1 can be more appropriate for applications.

\section{Application}

II-1. General frame. Consider the following integral equation:

$$
u(t)=\exp (-t) f+\int_{0}^{t} \exp (s-t) T u(s) d s,
$$

where:

i) $T: B \rightarrow B$ with $B$ a $\rho$-closed, convex subset of a Musielak-Orlicz space $L^{\varphi}$ satisfying the $\Delta_{2}$-condition.

ii) $T$ is $\rho$-Lipschitz:

$$
\exists \gamma>0, \rho(T u-T v) \leq \gamma \rho(u-v) u, v \in B .
$$

iii) $f \in B$.

Theorem II-1. Under these conditions, for all $A>0$ the integral equation (I) has a solution $u \in C^{\varphi}=C\left([0, A], L^{\varphi}\right) . C^{\varphi}$ is the modular space of continuous mappings from $[0, A]$ into $L^{\varphi}$

By iterative techniques, Khamsi [6] has shown this result under supplementary conditions: $B$ is $\rho$-bounded and $T$ is $\rho$-Lipschitz with constant $\gamma=1$.

To delete all restrictive assumptions on the Lipschitz constant $\gamma$ we introduce the space $C^{\varphi}$ equipped with a convenient modular.

Our method follows the standard technique of the resolution of differential equations or integral equations of Lipschitz type in Banach spaces. See Deimling [2, p. 39].

\section{II-2. Functional frame: The modular space $C^{\varphi}$.}

Definition 2-1. $u: I \rightarrow L^{\varphi}$, where $I=[0, A]$, is said to be continuous at $t_{0} \in I$ if: for $t_{n} \in I, t_{n} \rightarrow t_{0}$ as $n \rightarrow+\infty \Rightarrow \rho\left(u\left(t_{n}\right)-u\left(t_{0}\right)\right) \rightarrow 0$ as $n \rightarrow+\infty$.

Since $\rho$ satisfies the $\Delta_{2}$-condition, it is equivalent to:

$$
t_{n} \rightarrow t_{0} \text { as } n \rightarrow+\infty \Rightarrow\left|u\left(t_{n}\right)-u\left(t_{0}\right)\right|_{\rho} \rightarrow 0 \text { as } n \rightarrow+\infty \text {. }
$$

Let $C^{\varphi}=C\left(I, L^{\varphi}\right)$ be the space of all continuons mappings from $I=[0, A]$ into $L^{\varphi}$.

Proposition 2-1. Suppose that the modular $\rho$ satisfies $\left(\Delta_{2}\right)$, and $B \subset L^{\varphi}$ is a $\rho$-closed, convex subset of $L^{\varphi}$. For $a \geq 0$ let $\rho_{a}(u)=\sup _{t \in I} \exp (-a t) \rho(u(t))$ for $u \in C^{\varphi}$. Then:

1) $\left(C^{\varphi}, \rho_{a}\right)$ is a modular space, and $\rho_{a}$ is a convex modular satisfying the Fatou property and the $\Delta_{2}$-condition.

2) $C^{\varphi}$ is $\rho_{a}$-complete.

3) $C_{0}^{\varphi}=C(I, B)$ is a $\rho_{a}$-closed, convex subset of $C^{\varphi}$.

Proof. 1) i) $C^{\varphi}$ is a real vector space.

Let $u, v \in C^{\varphi}, t_{0} \in I$. Then for $t_{n} \in I$ such that $t_{n} \rightarrow t_{0}$ as $n \rightarrow+\infty$ we have:

$$
\rho\left(\frac{(u+v)\left(t_{n}\right)-(u+v)\left(t_{0}\right)}{2}\right) \leq \frac{1}{2} \rho\left(u\left(t_{n}\right)-u\left(t_{0}\right)\right)+\frac{1}{2} \rho\left(v\left(t_{n}\right)-v\left(t_{0}\right)\right) .
$$


Hence, $\rho\left(\frac{(u+v)\left(t_{n}\right)-(u+v)\left(t_{0}\right)}{2}\right) \rightarrow 0$ as $n \rightarrow+\infty$. And by $\Delta_{2}$ :

$$
\rho\left((u+v)\left(t_{n}\right)-(u+v)\left(t_{0}\right)\right) \rightarrow 0 \text { as } n \rightarrow+\infty,
$$

which implies that $(u+v)$ is continuous at $t_{0}$.

Again by $\Delta_{2} \rho\left(\lambda\left(u\left(t_{n}\right)-u\left(t_{0}\right)\right)\right) \rightarrow 0$ as $n \rightarrow+\infty$ for all $\lambda \in \mathbf{R}$; then $\lambda u$ is also continuous at $t_{0}$.

ii) $\rho_{a}$ is well defined.

Since $\rho$ satisfies the $\left(\Delta_{2}\right)$-condition, the domain of $\rho$ is $\left\{f \in L^{\varphi}, \rho(f)<+\infty\right\}=$

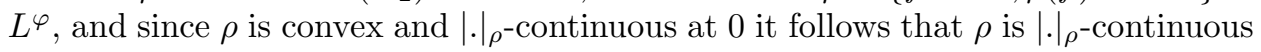
on $L^{\varphi}$. See Zeidler [9, p. 383].

Consequently for all $u \in C^{\varphi}, \rho_{a}(u)$ has a meaning.

iii) $\rho_{a}$ is a convex modular.

This is a simple consequence of the fact that $\rho$ is a convex modular.

iv) $\rho_{a}$ satisfies the Fatou property.

Let $\left(u_{n}\right)_{n}\left(\operatorname{resp}\left(v_{n}\right)_{n}\right)$ be a sequence in $C^{\varphi}, \rho_{a}$-convergent to $u$ (resp. to $\left.v\right) \in C^{\varphi}$. Then $\forall t \in I, \rho\left(u_{n}(t)-u(t)\right) \rightarrow 0$ as $n \rightarrow+\infty$ and $\rho\left(v_{n}(t)-v(t)\right) \rightarrow 0$ as $n \rightarrow+\infty$.

Since $\rho$ satisfies the Fatou property, we have:

$$
\begin{gathered}
\rho(u(t)-v(t)) \leq \liminf \rho\left(u_{n}(t)-v_{n}(t)\right) \forall t \in I, \\
\exp (-a t) \rho(u(t)-v(t)) \leq \liminf \exp (-a t) \rho\left(u_{n}(t)-v_{n}(t)\right) \leq \liminf \rho_{a}\left(u_{n}-v_{n}\right)
\end{gathered}
$$

and then

$$
\rho_{a}(u-v) \leq \liminf \rho_{a}\left(u_{n}-v_{n}\right) .
$$

v) $\rho_{a}$ has the $\left(\Delta_{2}\right)$-condition.

Since $\rho$ satisfies the $\left(\Delta_{2}\right)$-condition, one has:

$$
\begin{gathered}
\rho_{a}\left(u_{n}\right) \rightarrow 0 \text { as } n \rightarrow+\infty \Leftrightarrow \forall t \in I, \exp (-a t) \rho\left(u_{n}(t)\right) \rightarrow 0 \text { as } n \rightarrow+\infty \\
\Leftrightarrow \forall t \in I, \exp (-a t) \rho\left(2 u_{n}(t)\right) \rightarrow 0 \text { as } n \rightarrow+\infty \\
\Leftrightarrow \rho_{a}\left(2 u_{n}\right) \rightarrow 0 \text { as } n \rightarrow+\infty .
\end{gathered}
$$

2) It is known that $\left(L^{\varphi},|\cdot|_{\rho}\right)$ is a Banach space. Then the space $\left(C^{\varphi},|\cdot|_{\rho_{a}}\right)$ is also a Banach space. If $\left(u_{n}\right)_{n}$ is a $\rho_{a}$-Cauchy sequence in $C^{\varphi}$, by the $\Delta_{2}$-condition it is $|\cdot|_{\rho_{a}}$-Cauchy. Hence: $\exists u \in C^{\varphi}$ such that $u_{n} \rightarrow u$ in $\left(C^{\varphi},|\cdot|_{\rho_{a}}\right)$.

Consequently $\left(u_{n}\right) \rightarrow u$ in $\left(C^{\varphi}, \rho_{a}\right)$. It follows that $C^{\varphi}$ is $\rho_{a}$-complete.

3) $C_{0}^{\varphi}=C(I, B)$ is convex and $\rho_{a}$-closed.

The convexity of $C_{0}^{\phi}$ and its $\rho_{a}$-closedness are clearly obtained by the convexity and $\rho$-closedness of $B$ in $L^{\phi}$.

II-3. Proof of Theorem II-1. Define the operator $S$ over $C_{0}^{\varphi}$ by:

$$
\forall u \in C_{0}^{\varphi}, \quad S u(t)=\exp (-t) f+\int_{0}^{t} \exp (s-t) T u(s) d s, \quad \forall t \in I .
$$

$1^{\text {st }}$ step: We show that $S: C_{0}^{\varphi} \rightarrow C_{0}^{\varphi}$.

i) $S u$ is continuous from $I$ into $\left(L^{\varphi},|\cdot|_{\rho}\right)$.

Let $t_{n}, t_{0} \in I$ with $t_{n} \rightarrow t_{0}$ as $n \rightarrow+\infty$.

$T$ is $\rho$-Lipschitz $\rho\left(T u\left(t_{n}\right)-T u\left(t_{0}\right)\right) \leq \gamma \rho\left(u\left(t_{n}\right)-u\left(t_{0}\right)\right)$. Since $u$ is $\rho$-continuous, $T u$ is $\rho$-continuous at $t_{0}$ and by $\left(\Delta_{2}\right), T u$ is $|\cdot|_{\rho}$-continuous at $t_{0}$. Hence $S u$ is $|\cdot|_{\rho^{-}}$ continuous at $t_{0}$.

ii) $S u(t) \in B, \quad \forall t \in I$. 
It is well known that in the Banach space $\left(L^{\varphi},|\cdot|_{\rho}\right)$

$$
\begin{aligned}
\int_{0}^{t} \exp (s-t) T u(s) d s & \in\left(\int_{0}^{t} \exp (s-t) d s\right) \overline{c o}\{T u(s), 0 \leq s \leq t\} \\
& \in(1-\exp (-t)) \overline{c o} B
\end{aligned}
$$

where $\overline{c o} B$ is the closed convex hull of $B$ in $\left(L^{\varphi},|\cdot|_{\rho}\right)$. But $B$ is convex and $\rho$-closed; thus, $\overline{c o} B=\bar{B} \subset \overline{B^{\rho}}=B$. Hence, $S u(t) \in \exp (-t) B+(1-\exp (-t)) B \subseteq B \quad \forall t \in$ I.

$2^{\text {nd }}$ step: For $u, v \in C_{0}^{\varphi}$ and $\lambda>0$ we have:

$$
\lambda(S u(t)-S v(t))=\int_{0}^{t} \lambda \exp (s-t)(T u(s)-T v(s)) d s .
$$

Lemma 3-1 ([6]). Let $x \in C^{\varphi}$ and $0<\lambda \leq \frac{\exp A}{\exp (A)-1}$. Then:

$$
\rho\left(\int_{0}^{t} \lambda \exp (s-t) x(s) d s\right) \leq \lambda \frac{\exp (a t)-\exp (-t)}{1+a} \rho_{a}(x) .
$$

Note that in [6] this result was been shown with $\lambda=1$ and $a=0$.

Proof. Let $T=\left\{t_{0}, t_{1}, t_{2}, \ldots, t_{n}\right\}$ be any subdivision of $[0, t]$.

$$
\sum_{i=0}^{n-1} \lambda\left(t_{i+1}-t_{i}\right) \exp \left(t_{i}-t\right) x\left(t_{i}\right)
$$

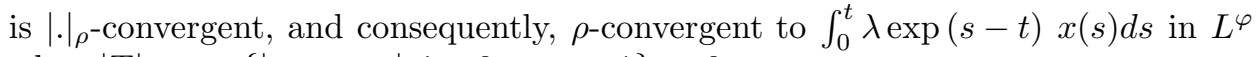
when $|T|=\sup \left\{\left|t_{i+1}-t_{i}\right|, i=0, \ldots, n-1\right\} \rightarrow 0$ as $n \rightarrow+\infty$.

By Fatou we have:

$$
\rho\left(\int_{0}^{t} \lambda \exp (s-t) x(s) d s\right) \leq \liminf \rho\left(\sum_{i=0}^{n-1} \lambda\left(t_{i+1}-t_{i}\right) \exp \left(t_{i}-t\right) x\left(t_{i}\right)\right) .
$$

On the other hand:

$$
\begin{aligned}
\sum_{i=0}^{n-1} \lambda\left(t_{i+1}-t_{i}\right) \exp \left(t_{i}-t\right) & \leq \int_{0}^{t} \lambda \exp (s-t) d s \\
& =(1-\exp (-t)) \lambda \leq \lambda(1-\exp (-A)) .
\end{aligned}
$$

Since $0<\lambda<\frac{\exp (A)}{\exp (A)-1}$, it follows that $\sum_{i=0}^{n-1} \lambda\left(t_{i+1}-t_{i}\right) \exp \left(t_{i}-t\right) \leq 1$, and the convexity of $\rho$ implies:

$$
\begin{aligned}
& \rho\left(\sum_{i=0}^{n-1} \lambda\left(t_{i+1}-t_{i}\right) \exp \left(t_{i}-t\right) x\left(t_{i}\right)\right) \leq \sum_{i=0}^{n-1} \lambda\left(t_{i+1}-t_{i}\right) \exp \left(t_{i}-t\right) \rho\left(x\left(t_{i}\right)\right) \\
& \quad=\sum_{i=0}^{n-1} \lambda\left(t_{i+1}-t_{i}\right) \exp \left(t_{i}-t\right) \exp \left(a t_{i}\right) \exp \left(-a t_{i}\right) \rho\left(x\left(t_{i}\right)\right) \\
& \quad \leq \sum_{i=0}^{n-1} \lambda\left(t_{i+1}-t_{i}\right) \exp \left((1+a) t_{i}-t\right) \rho_{a}(x) \\
& \quad \leq\left(\int_{0}^{t} \lambda \exp ((1+a) s-t) d s\right) \rho_{a}(x) .
\end{aligned}
$$


Then:

$$
\begin{aligned}
& \quad \rho\left(\int_{0}^{t} \lambda \exp (s-t) x(s) d s\right) \leq \lambda \frac{\exp (a t)-\exp (-t)}{1+a} \rho_{a}(x) . \\
& 3^{r d} \text { step: } \\
& \rho(\lambda(S u(t)-S v(t))) \leq \lambda \frac{\exp (a t)-\exp (-t)}{1+a} \rho_{a}(T u-T v) .
\end{aligned}
$$

But

$$
\rho_{a}(T u-T v)=\sup _{t \in I} \exp (-a t) \rho(T u(t)-T v(t)) \leq \gamma \rho_{a}(u-v)
$$

Then:

$$
\begin{aligned}
\exp (-a t) \rho(\lambda(S u(t)-S v(t)) & \leq \lambda \frac{\gamma}{1+a}(1-\exp ((-t)(1+a))) \rho_{a}(u-v) \\
& \leq \lambda \frac{\gamma}{1+a}(1-\exp ((-A)(1+a))) \rho_{a}(u-v), \quad \forall t \in I
\end{aligned}
$$

Hence

$$
\rho_{a}(\lambda(S u-S v)) \leq \lambda \frac{\gamma}{1+a}(1-\exp ((-A)(1+a))) \rho_{a}(u-v)
$$

We consider $\lambda, 1<\lambda \leq \frac{\exp (A)}{\exp (A)-1}$. Then $S$ has a fixed point if:

$$
\lambda \frac{\gamma}{1+a}(1-\exp ((-A)(1+a)))<\lambda \Leftrightarrow \frac{\gamma}{1+a}(1-\exp ((-A)(1+a)))<1
$$

The last inequality is satisfied if we take for example $a \geq \gamma$.

In the end, by Theorem I-1, $S$ has a fixed point which is a solution of the integral equation (I).

\section{REFERENCES}

[1] A. Ait Taleb, Points fixes et Applications aux equations integrales dans les espaces modulaires. These de 3me cycle, Departement de Mathematiques et Informatique, Rabat (1996).

[2] K. Deimling, Nonlinear Functional Analysis, Springer Verlag, (1985). MR 86j:47001

[3] K. Goebel-S. Reich, Uniform convexity, Hyperbolic Geometry and nonexpansive mappings, Dekker, (1984). MR 86d:58012

[4] K. Goebel-W.A. Kirk, Topics in metric fixed point theory, Cambrige University Press, Cambridge (1990). MR 92c:47070

[5] M.A. Khamsi-W.M. Kozlowski-S. Reich, Fixed point theory in modular Function spaces. Nonlinear Analysis, theory, methods and Applications. vol.14. $N^{0} 11$ (1990). 935-953. MR 91d:47042

[6] M.A. Khamsi, Nonlinear semigroups in modular Function space thèse d' état. Departement de Mathmatiques, Rabat (1994). MR 93g:47085

[7] W.M. Kozlowski, Modular function spaces. Dekker (1988). CMP 98:02

[8] J. Musielak, Orlicz spaces and modular spaces, Lecture notes in Mathematics, vol 1034, Springer Verlag (1983). MR 85m:46028

[9] E. Zeidler, Nonlinear Functional Analysis and its Applications tome III, Springer Verlag (1985). MR 90b:49005

Department of Mathematics and Informatic, Faculte des Sciences, Universite Mohammed V, BP. 1014, Rabat, Morocco 